EUROPEAN ORGANIZATION FOR NUCLEAR RESEARCH

European Laboratory for Particle Physics

LHC Project Report 143

Large Hadron Collider Project

\title{
A Simplified Cryogenic Distribution Scheme for the Large Hadron Collider
}

M. Chorowski, W. Erdt, Ph. Lebrun, G. Riddone, L. Serio,

L. Tavian, U. Wagner, and R. van Weelderen

\begin{abstract}
The Large Hadron Collider (LHC), currently under construction at CERN, will make use of superconducting magnets operating in superfluid helium below $2 \mathrm{~K}$. The reference cryogenic distribution scheme was based, in each $3.3 \mathrm{~km}$ sector served by a cryogenic plant, on a separate cryogenic distribution line which feeds elementary cooling loops corresponding to the length of a half-cell $(53 \mathrm{~m})$. In order to decrease the number of active components, cryogenic modules and jumper connections between distribution line and magnet strings a simplified cryogenic scheme is now implemented, based on cooling loops corresponding to the length of a full-cell $(107 \mathrm{~m})$ and compatible with the LHC requirements. Performance and redundancy limitations are discussed with respect to the previous scheme and balanced against potential cost savings.
\end{abstract}

* LHC Division

Administrative Secretariat

LHC Division

CERN

CH - 1211 Geneva 23

Switzerland

Geneva, 29 September 1997 


\title{
A SIMPLIFIED CRYOGENIC DISTRIBUTION SCHEME FOR THE LARGE HADRON COLLIDER
}

\author{
M. Chorowski, W. Erdt, Ph. Lebrun, G. Riddone, L. Serio, \\ L. Tavian, U. Wagner, and R. van Weelderen \\ LHC Division, CERN, CH-1211 Geneva 23, Switzerland
}

\begin{abstract}
The Large Hadron Collider (LHC), currently under construction at CERN, will make use of superconducting magnets operating in superfluid helium below $2 \mathrm{~K}$. The reference cryogenic distribution scheme was based, in each $3.3 \mathrm{~km}$ sector served by a cryogenic plant, on a separate cryogenic distribution line which feeds elementary cooling loops corresponding to the length of a half-cell $(53 \mathrm{~m})$. In order to decrease the number of active components, cryogenic modules and jumper connections between distribution line and magnet strings a simplified cryogenic scheme is now implemented, based on cooling loops corresponding to the length of a full-cell $(107 \mathrm{~m})$ and compatible with the LHC requirements. Performance and redundancy limitations are discussed with respect to the previous scheme and balanced against potential cost savings.
\end{abstract}

\section{INTRODUCTION}

In the former conceptual design of the $\mathrm{LHC}^{1}$, the cryogenic distribution is based on a four-point feed scheme, with eight cryogenic plants distributed in pairs at each even point and each dedicated to the cooling of a complete $3.3-\mathrm{km}$ sector. Within a sector, a separate cryogenic line feeds elementary cooling loops corresponding to the length of a half-cell $(53 \mathrm{~m})$. For a maximum of standardization, the layout of functional components (bus-bar plugs, cooldown-and-fill valves, safety relief valves, 1.8-K cooling loop components, etc.) in all elementary cooling loops is identical. The subcooling of helium for the superfluid helium cooling loop is centralized in the cold compressor boxes and its distribution requires a dedicated header for liquid helium at $2.2 \mathrm{~K}$ and $0.13 \mathrm{MPa}$.

The optimization of the distribution scheme by rearranging the cryogenic plant location and reducing the number of components and distribution headers as well as by increasing the cooling loop length to a full-cell, leads to major cost savings, but forces us to abandon the concept of complete standardization. 


\section{CRYOGENIC ARCHITECTURE}

In view of installation and space constraints, particularly in underground caverns, and following a technical-economical study ${ }^{2}$, the general architecture of the cryogenic system ${ }^{3}$ has been modified. The four new cryogenic plants, which have to be added to the four existing split-coldbox helium refrigerators of LEP, are now integrally located at surface level. In addition, the decision of grouping two cryogenic plants at each even point was overruled for point 2 which will only keep the upgraded LEP refrigerator, since the other cryogenic plant is now foreseen in the area 18 of the point 1 . Figures 1 and 2 show the location and the new architecture of the LHC cryogenics.

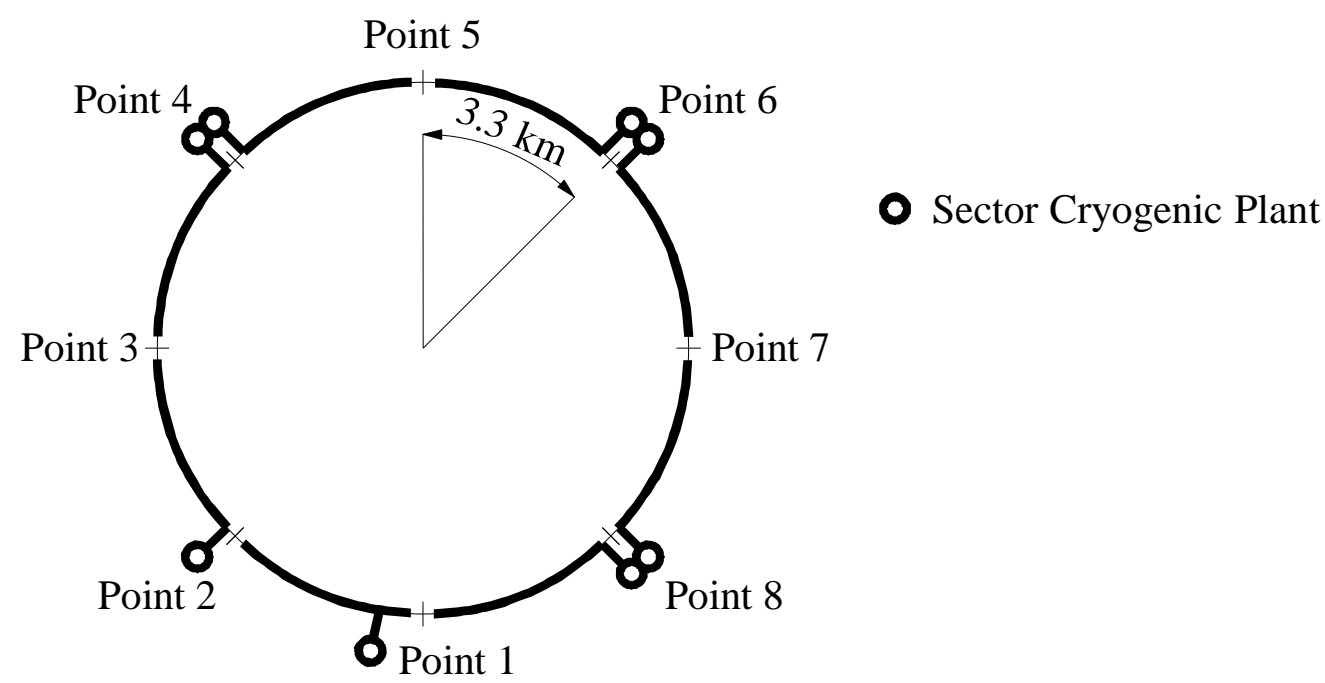

Figure 1. Location of LHC cryogenic plants

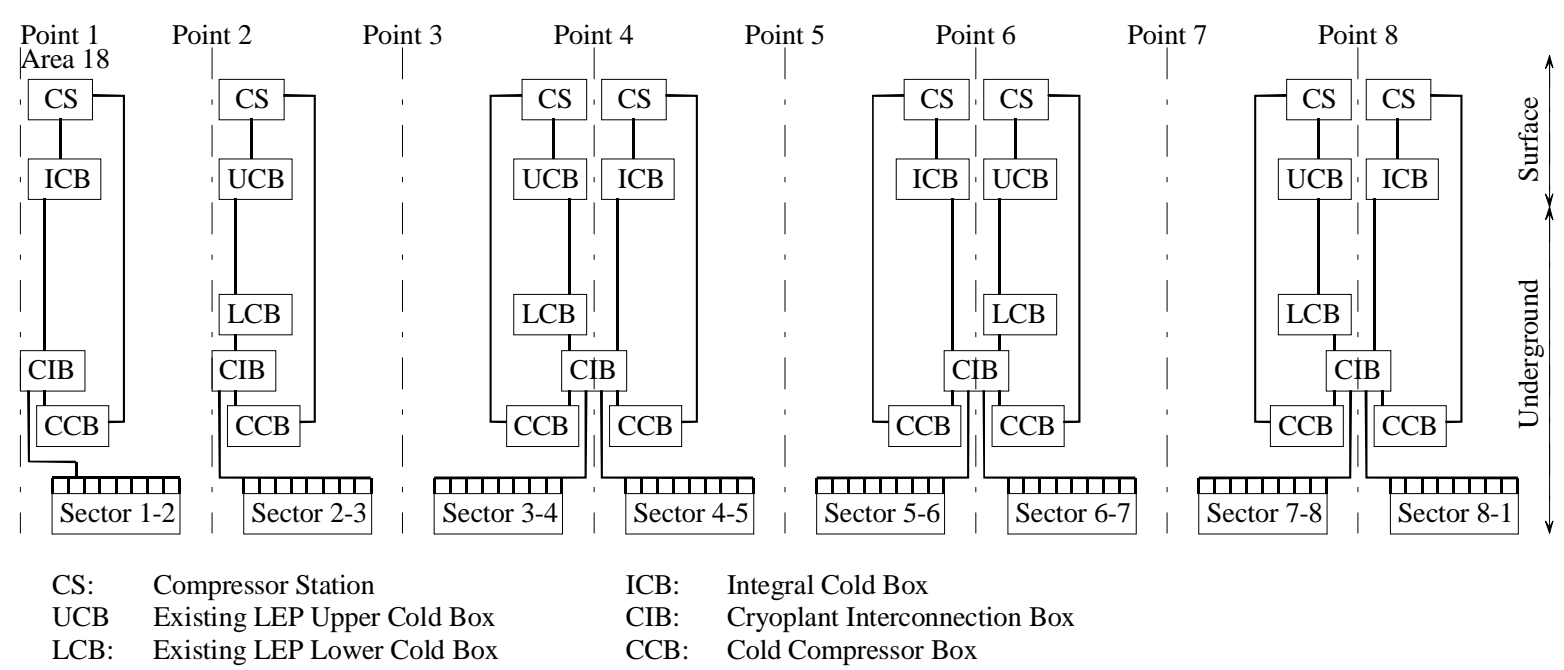

Figure 2. Cryogenic architecture of LHC

As a consequence of this new architecture, the cryogenic plant at point 1 , located at the lowest point of the sector 1-2 gives a better cooling performance of this sector with respect to the previous configuration. In point 1 , the cryogenic plant will first be used as a source of liquid helium for component tests. However, the interconnection between two neighbouring plants is no longer possible at point 2. 


\section{REDUCING THE NUMBER OF DISTRIBUTION HEADERS}

In order to reduce the vapour mass fraction produced in the Joule-Thomson expansion of the superfluid cooling loop, a subcooled helium supply is required. The subcooling can be performed centrally in the cold compressor box of the corresponding sector, or distributed in each local cooling loop. The first case requires a single heat exchanger for the total flow of about $120 \mathrm{~g} / \mathrm{s}$ and a distribution header in the cryogenic distribution line. In the second case, the need for this header disappears, but one smaller heat exchanger is needed for each cooling loop. The major difference between these two cases is the temperature of the returning low-pressure vapour in the pumping header, which has opposite consequences on the hydrostatic and frictional terms of the pressure drop. In the first case (central heat exchanger), the very-low-pressure vapour is pumped at $1.8 \mathrm{~K}$, when it is dense, which minimizes frictional pressure drop. In the second case (distributed heat exchanger), the very-low-pressure helium is returned to the $\mathrm{CCB}$ at higher temperature, thus reducing hydrostatic head. Figure 3 shows the magnet temperature profiles which directly depend on the pressure profile of the pumping line, for nominal operating conditions.

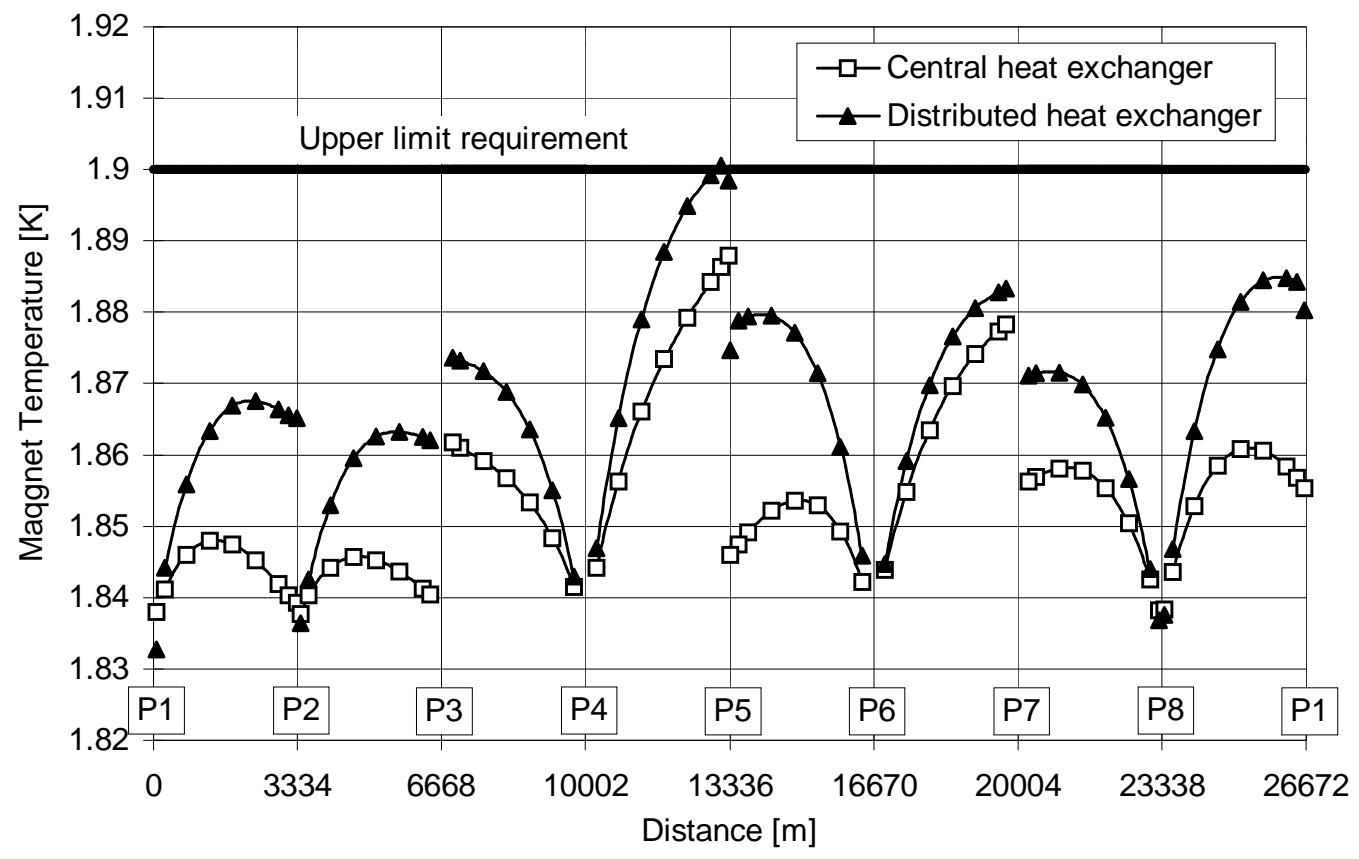

Figure 3. Magnet temperature profiles at nominal conditions (with uncertainty factor)

With distributed heat exchangers, the maximum magnet temperature is slightly higher $(10 \mathrm{mK})$ but is still below the upper limit requirement of $1.9 \mathrm{~K}$. A distribution scheme using distributed heat exchangers is acceptable. In addition, the validation of the design and construction of small distributed heat exchangers for a flow of about $5 \mathrm{~g} / \mathrm{s}$, is easier.

\section{REDUCING THE NUMBER OF COMPONENTS}

The optimization of the cryogenic distribution scheme by reducing the number of components will decrease costs and heat inleaks, and increase the reliability of the cryogenic system. 


\section{Bus-Bar Plugs}

The bus-bar plugs segment the pressurized helium volume in the cold mass, which contains the magnets and the electrical bus bars connecting them in series. This hydraulic segmentation is required for the following reasons:

- limitation of the hydrostatic head due to the tunnel slope: the cold mass must remain above the atmospheric pressure to prevent air inleaks into circuits,

- possibility of short interventions on the cold mass (e.g. exchange of diodes, repair of instrument feedthrough, etc.); for such interventions, the warmup and cooldown time must be minimized and are only limited by the maximum allowed flow-rate per cell,

- possibility of magnet removal which require a sub-sectorization ${ }^{4}$ of the sector.

The previous design was based on one segmentation at every half-cell, i.e. 53 bus-bar plugs per sector. Based on the above requirements, the number of bus bar plugs can be reduced to 14 elements per sector. Table 1 gives the bus-bar plug requirement for the previous and simplified schemes. Figure 4 shows the bus-bar plug spacing for the simplified scheme.

Table 1. Requirements of components number

\begin{tabular}{lcc}
\hline Component Type & Previous scheme & Simplified scheme \\
\hline Bus-bar plug & 53 & 14 \\
Cooldown and fill valve & 54 & 15 \\
Safety relief valve & 108 & 42 \\
\hline
\end{tabular}

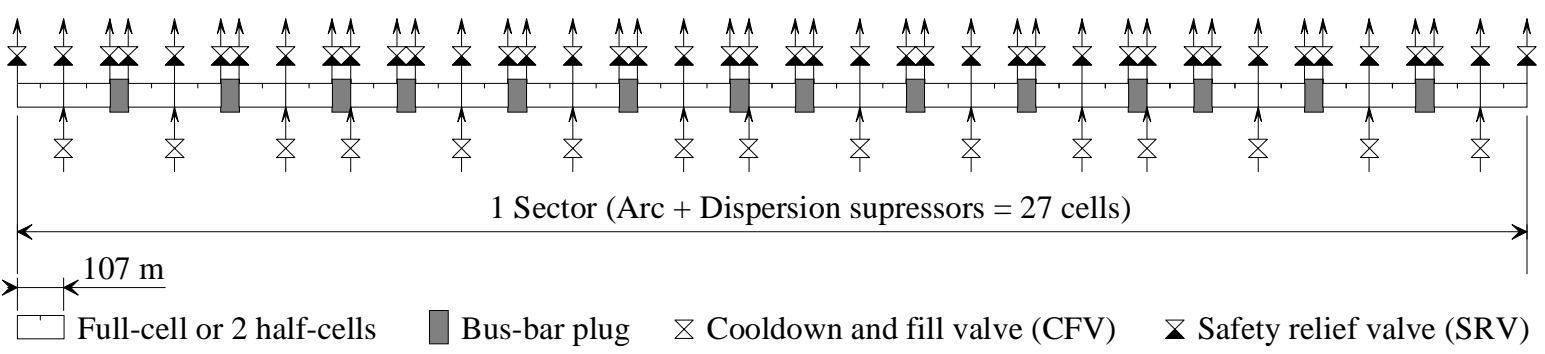

Figure 4. Requirement of component spacing of the simplified scheme

\section{Cooldown-and-Fill Valves (CFV)}

Due to pressure drop limitations, a maximum string length, corresponding to one fullcell, is acceptable for cooldown. With the bus-bar spacing defined for the simplified scheme and by using parallel cooling of two full-cells adjacent to a cooldown-and-fill valve, it is possible to cool down and warm up the sector without adding other bus-bar plugs between cells. Consequently, with respect to the previous scheme, the number of valves can be reduced from 54 down to 15 (see Table 1 and Figure 4). For parallel cooling, the flow balance and the cooldown time depend on differences in mass and hydraulic impedance between the two adjacent cells. Possible unbalanced cooldown could be corrected by pulseduration modulation type control on the outlet valves. 
The lengthening of the cooling loop to a full-cell increases the minimum distance between two safety relief valves from 53 up to $107 \mathrm{~m}$. Figure 5 shows simulations of fullquench discharges of a cell for normal protection (simultaneous discharge through two SRV's) and for an accidental case in which one of these safety relief valve remains closed. These simulations were performed using a numerical model ${ }^{5,6}$ based on quench discharges measurements of the Test String, in operation at CERN. The normal protection of the simplified scheme has been validated on the $50-\mathrm{m}$ Test String by discharging it at one of its extremities only.

During a full-cell quench, the maximum pressure in the cold mass evolves around 1.3 $\mathrm{MPa}$, which represents an ample safety margin with respect to the 2-MPa cold-mass design pressure. The number and spacing of SRV's, given in Figure 4, therefore conforms with the simplified scheme.
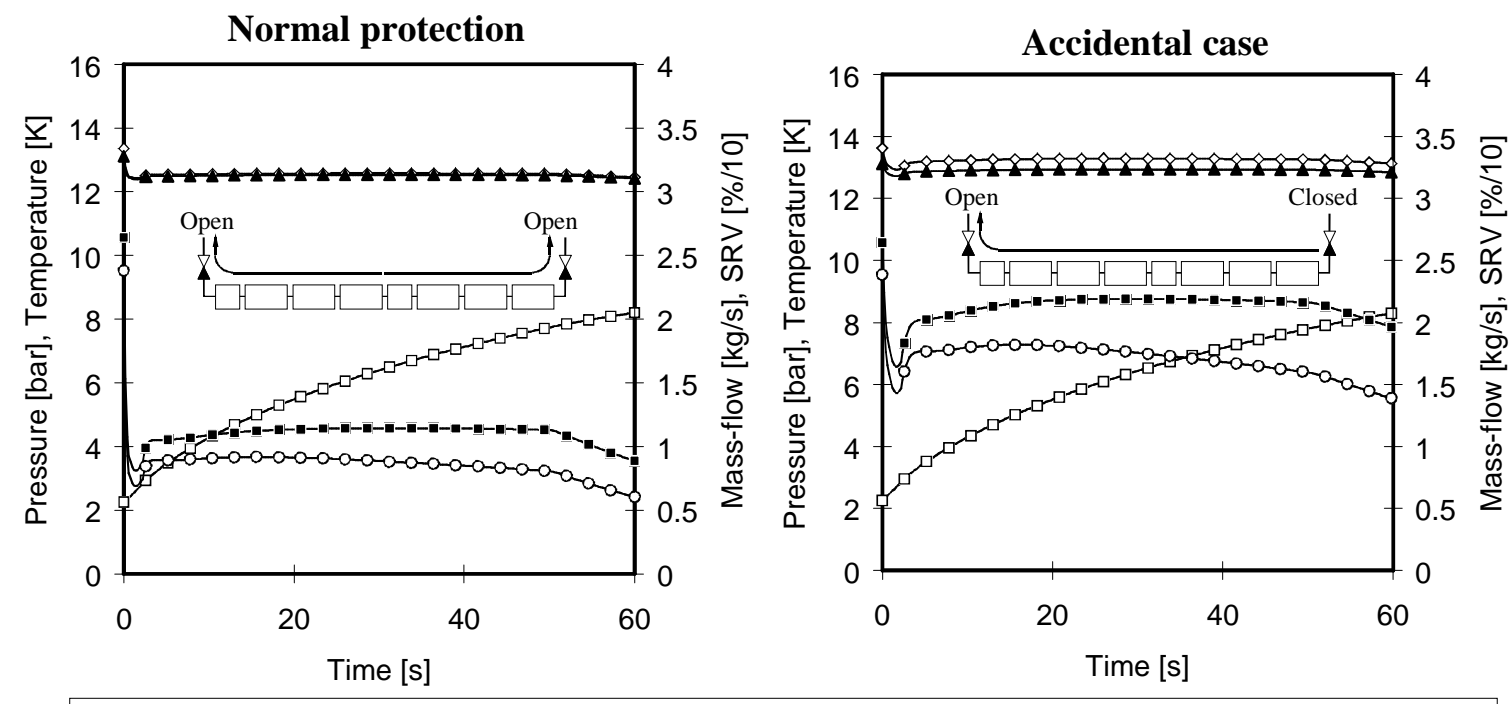

$\rightarrow-$ Temperature $\rightarrow$ Maximum pressure $\leftarrow$ SRV Inlet pressure $\rightarrow-$ Mass-flow - - SRV opening

Figure 5. Simulations of quench discharge of full-cells.

\section{INCREASE OF THE LENGTH OF ELEMENTARY COOLING LOOPS}

The component spacing defined above provides the possibility to increase the length of the elementary cooling loops to $107 \mathrm{~m}$, and consequently reduce the number of cryogenic modules feeding the superconducting magnets from the cryogenic distribution line. To achieve this, two local loops, corresponding to the cold mass and the beam screen cooling, have to be modified.

\section{Supercritical Helium Beam-Screen Cooling Loop}

The pressure drop in this circuit due to the very high hydraulic impedance of the beam screen channels does not allow the increase of the loop length to $107 \mathrm{~m}$. Left-right supply is the only way compatible with a larger spacing between cryogenic modules. Figure 7 shows the new arrangement of the beam-screen cooling loops with two outlet temperature control valves (TCV2) per cryogenic module. 


\section{Superfluid Helium Cold-Mass Cooling Loops}

Saturated liquid helium in the cold-mass heat exchanger always flows downslope. Consequently, left-right supply circuits are not a viable option for the cold-mass cooling loops, which therefore need to be doubled in length.

The thermal performance of the cold mass heat exchanger ${ }^{8}$ depends partly on its pressure drop. For given pipe geometry and linear heat load, doubling the length of this heat exchanger would lead to an increase in the corresponding pressure drop by a factor 8 which is unacceptable for the magnet cooling. The length increase must thus be compensated by modifying the pipe geometry using a combination of a change of friction factor and of hydraulic diameter. The outer diameter of the heat exchanger is limited by the $60 \mathrm{~mm}$ diameter aperture in the magnet yoke. Figure 6 shows two heat exchanger cross-sections as well as their main characteristics for the previous and simplified schemes. Taking into account the higher pressure level in the supply header, the external diameter of the inner pipe can be reduced from $14 \mathrm{~mm}$ to $12 \mathrm{~mm}$. For the simplified scheme, the friction factor is improved by a factor 4 and the hydraulic diameter by a factor 1.2, the combination of which gives a pressure reduction by a factor 10 . The hydraulic performance of the simplified scheme heat exchanger is better than that of the previous one.

The thermal and mechanical performance is determined by the wall thickness, which must be mechanically designed to withstand an external over-pressure of $2 \mathrm{MPa}$, while being as thin as possible for limiting transverse thermal impedance. The best thermomechanical optimization is to use OFHC copper with $20 \%$ of cold work which increases the mechanical properties and requires a wall thickness of $2 \mathrm{~mm}$ only. The use of bellows may be required in each cold mass to compensate for thermal expansion.

The cooling loop length of $107 \mathrm{~m}$ allows the number of expansion valves TCV1 to be halved. The decision was made to add spare valves (TCV1') parallel to the expansion valves TCV1 and their subcooling heat exchangers. Such an arrangement permits a faster cooldown of the subcooling heat exchanger, a greater dynamic range for faster recooling after a magnet quench, and provides redundancy of the cooling loop in case of TCV1 valve failure or subcooling heat exchanger plugging.

\begin{tabular}{|c|c|}
\hline Previous Scheme Type: & Simplified Scheme Type: \\
\hline $\begin{array}{l}\text { Characteristics: } \\
\text { Corrugated, } \\
\text { DHP Copper } \\
\text { Dimensions: } 49 \times 57 \mathrm{~mm} \text {, } \\
\text { Thickness } 1 \mathrm{~mm} \\
\text { Inner pipe: } 12 \times 14 \mathrm{~mm} \\
\text { Hydraulic diameter: } 35 \mathrm{~mm} \\
\text { Friction factor: } 0.08\end{array}$ & $\begin{array}{l}\text { Characteristics: } \\
\text { Cylindrical } \\
\text { OFHC Copper } \\
20 \% \text { of cold work } \\
\text { Dimensions: } 54 \times 58 \mathrm{~mm}, \\
\text { Thickness: } 2 \mathrm{~mm} \\
\text { Inner pipe: } 10 \times 12 \mathrm{~mm} \\
\text { Hydraulic diameter: } 42 \mathrm{~mm} \\
\text { Friction factor: } 0.02\end{array}$ \\
\hline
\end{tabular}

Figure 6. Cold mass heat exchanger cross sections and characteristics.

\section{THE SIMPLIFIED DISTRIBUTION SCHEME}

Figure 7 shows the simplified scheme of the cryogenic distribution, which takes into account the component spacing and the cooling loop modifications. 


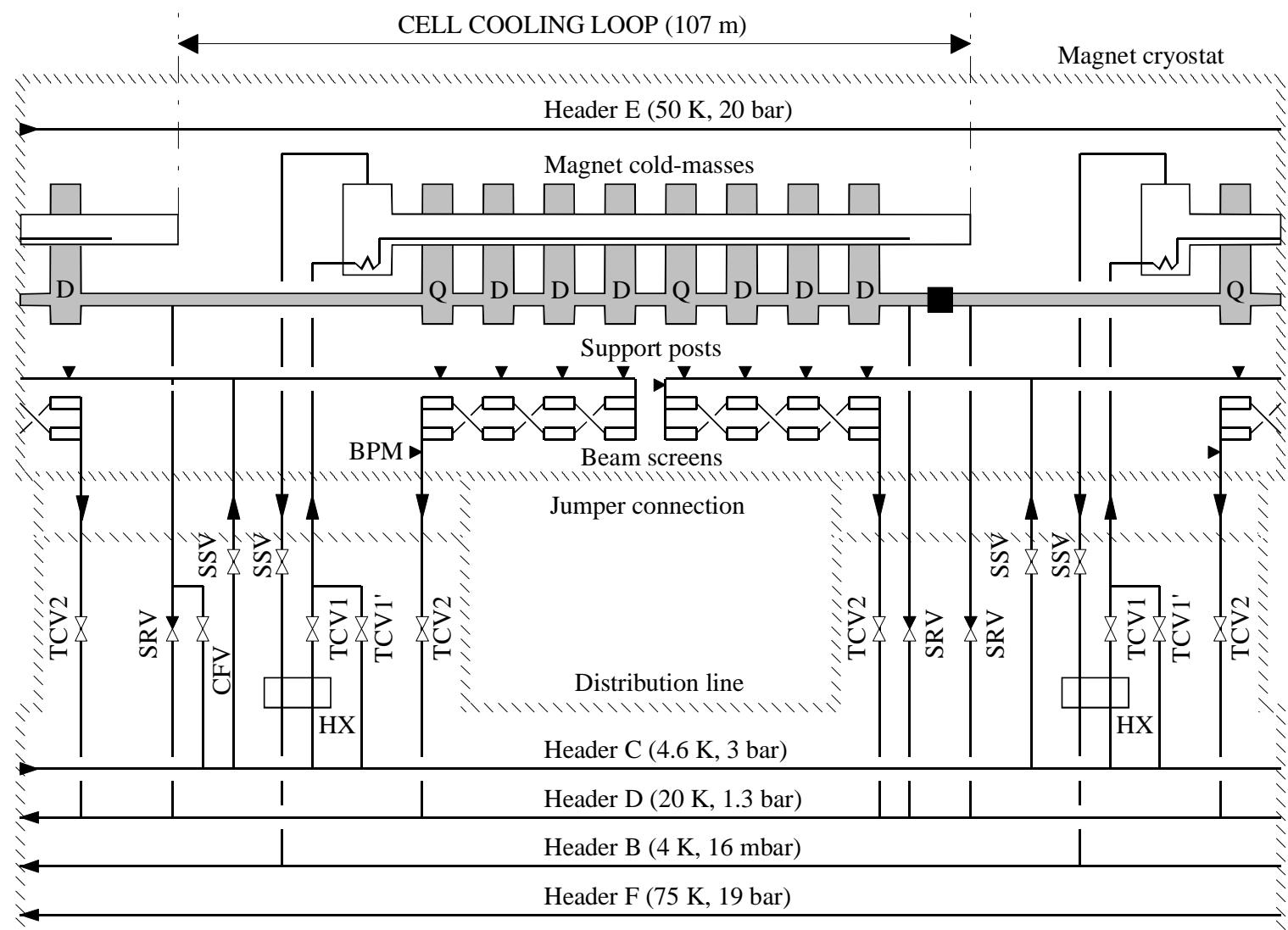

Figure 7. Simplified distribution scheme.

\section{COMPONENT INVENTORY AND COST SAVINGS}

Table 2 gives the variation of component numbers with respect to the previous scheme for the complete machine, yielding a total reduction of 1260 valves, 312 bus-bar plugs and 216 cryogenic modules. The reduction of the number of components increases the reliability of the cryogenic system and gives corresponding cost savings of about $20 \mathrm{M} \$$. The cost of extra subcooling heat exchangers is largely compensated by the cost saving corresponding to the suppression of the distribution header of subcooled liquid.

Table 2. Component requirements and variation with respect to the previous scheme

\begin{tabular}{lcc}
\hline Component type & Number/type & Variation \\
\hline Distribution header & 5 & -1 \\
Subcooling heat exchanger & 231 & +223 \\
Cryogenic module & 236 & -216 \\
Bus bar plug & 124 & -312 \\
Phase separator sump & 227 & -217 \\
Control valve & 1028 & -300 \\
Safety relief valve & 360 & -528 \\
Sub-sector valve & 456 & -432 \\
\hline
\end{tabular}




\section{CONCLUSION}

The simplification of the cryogenic architecture is based on a four-plus-one feed scheme using four new cryogenic plants located integrally at ground level surface in addition to the four existing LEP2 plants. This new architecture results from a technicaleconomical optimization taking into account the cooling requirement of LHC and the high cost of cavern excavation.

The simplification of the cryogenic distribution scheme is based on the reduction in the number of active components and of distribution headers with the suppression of subcooled liquid distribution and the addition of distributed subcooling heat exchangers. These simplifications improve the reliability of the cryogenic system, preserve the operation and safety requirements, reduce heat inleaks and increase the redundancy level of the superfluid helium cold-mass cooling loop. The improvements far outweigh the inconvenience of some loss in standardization and in addition represent a cost reduction of about $20 \mathrm{M}$.

\section{ACKNOWLEDGEMENT}

We would like to acknowledge the contributions of our colleagues J. Casas-Cubillos, B. Hilbert, T. Kurtyka, P. Rohmig, F. Savary and B. Skoczen of CERN.

\section{REFERENCES}

1. The LHC Study Group, “The Large Hadron Collider, Conceptual Design,” CERN Report AC/95-05(LHC) (1995).

2. U. Wagner, "The LHC refrigerators with surface located cold boxes for the temperature range 300-4.5 K," LHC Project Note 70 (1996).

3. V. Benda, A. Bézaguet, J. Casas-Cubillos, S. Claudet, W. Erdt, Ph. Lebrun, G. Riddone, V. Sergo, L. Serio, L. Tavian, B. Vuillerme, R. van Weelderen and U. Wagner, Conceptual design of the cryogenic system for the Large Hadron Collider, in: "Proceeding of EPAC96", S. Meyers, A. Pacheco, R. Pascual, Ch. Petit-Jean-Genaz and J. Poole, editors, Institute of Physics Publishing, Bristol and Philadelphia (1996), p. 361-363.

4. M. Bona, P. Cruikshank, W. Erdt, J.L. Périnet-Marquet, A. Poncet, P. Rohmig, U. Wagner and T. Wikberg, "Cryogenic and Vacuum Sectorization of the LHC Arcs," LHC Project Report 60 (1996)

5. B. Hilbert, "Modeling and Experimental Analysis of the Thermohydraulics of Resistive Transitions on the LHC Prototype Magnet String," LHC Project Note 86 (1997).

6. M. Chorowski, B. Hilbert, L. Serio and R. van Weelderen, Thermohydraulics of Resistive Transitions of the LHC Prototype Magnet String: Theoretical Modelling and Experimental Results, paper presented at this conference.

7. A. Bézaguet et al, The LHC Test String: First Operational Experience, in: "Proceeding of EPAC96", S. Meyers, A. Pacheco, R. Pascual, Ch. Petit-Jean-Genaz and J. Poole, editors, Institute of Physics Publishing, Bristol and Philadelphia (1996), p. 358-360.

8. Ph. Lebrun, L. Serio, L. Tavian and R. van Weelderen, Cooling Strings of Superconducting device below 2 K: The Helium II Bayonet Heat Exchanger, paper presented at this conference. 